\title{
Infección Parasitaria Asintomática en Lactantes Menores de 6 Meses
}

\author{
Dr. Julio Espinoza M., T.M. Ana María Altieri S., T.M. lsolda Pacheco M., E.U. Sara Labrin F., \\ Dra. Magdalena Araya Q, Dr. Oscar Brunser T.1.
}

\author{
Asymptomatic Parasitic Infections \\ in Infants
}

\begin{abstract}
Asymptomatic parasitic infection and presence of commensals was evaluated in 207 breast-weaned infants under 6 month of age and their relatives. Parasites (G. Lamblia, E. histolytica, and A. lumbricoides) were identified in $4.8 \%$ of the infants and in $14 \%$ of their relatives. No correlation between nutritional status and parasitic unfection was detected. All infants had a relative who was harbouring the same parasite.

(Key words: Asymptomatic intestinal infection. Ascaris lumbricotdes. Entamoeba histolytica. Giardia lamblia. Parasitic infection).
\end{abstract}

La portación de parásitos es un fenómeno frecuente en individuos que viven en ambientes mal saneados ${ }^{1-2}$. Estudios previos demuestran que hasta un $60 \%$ de escolares ${ }^{3}$ y $68.8 \%$ de pre-escolares ${ }^{4}$ residentes en áreas periurbanas de Santiago de estrato socioeconómico bajo, son portadores asintomáticos de enteroparásitos. Giardia lamblia y Entamoeba histolytica son los que se detectan con mayor frecuencia. Ambos agentes microbiológicos pueden asociarse a diarrea aguda e influenciaron negativamente el estado nutricional ${ }^{5-t}$.

Los Estudios de portación parasitaria en lactantes son escasos y las incidencias publicadas oscilan entre 20 y $26 \%{ }^{7-8}$. En estudios realiza. dos por nosotros, sobre infección asintomática bacteriana, las tasas de aislamiento son altas durante el primer año de vida $18.6 \%$ \%

1. Unidad de Gastroenterologia, Instituto de Nutrición y Tecnología de los Alimentos, (INTA) Universidad de Chile.
El objetivo de esta investigación es determinar la frecuencia de enteroparásitos en lactantes menores de 6 meses, aparentemente sanos, de nivel socioeconómico bajo y correlacionar estos resul. tados con la frecuencia de portadores de parásitos en su grupo familiar.

\section{SUJETOS Y PROCEDIMIENTOS}

El estudio de portación de parásitos se efectuó en 207 familias residentes en un área periurbana del sector Oriente de Santiago que fueron contac. tadas en sus casas a través del Policlínico La Faena.

Este protocolo fue aprobado por la Comisión de Etica del Instituto de Nutrición y Tecnología de los Alimentos y en consecuencia, la participación de los sujetos contó con el consentimiento por escrito de los adultos y de los padres o guardianes legales en el caso de los lactantes.

Los lactantes incluidos en esta investigación debian cumplir los siguientes requisitos: peso de 
nacimiento igual o superior a los $2.500 \mathrm{grs}$, haber dejado el pecho materno espontáneamente por lo menos 15 dias antes del inicio de este protocolo, examen físico nomal, sin antecedentes o evidencias de afecciones crónicas, estado nutricional normal o desnutrido leve de acuerdo con las Tablas del "National Center for Health Statistics" (NCHS) ${ }^{10}$ y pertenecer al nivel socioeconómico bajo de acuerdo con la escala de Graffar modificada para este tipo de poblaciones ${ }^{11}$. Además, no debían haber presentado un episodio de diarrea o recibido tratamiento antibiótico ni quimioterápico a lo menos 15 días antes $y$ después de iniciarse la investigación.

El estudio parasitológico de deposiciones se hizo en 3 muestras fecales obtenidas dia por medio y procesadas siguiendo la técnica de Burrows ${ }^{12}$. Conocido el resultado del examen coproparasitario se estudiaron los grupos familiares de los lactantes infectados.

\section{RESULTADOS}

La Tabla 1 muestra el número y la proporción de exámenes coproparasitarios que demostcaron la presencia de parásitos o comensales.

Las especies de parásitos y comensales encontrados se describen en la Tabla 2.

Los resultados del examen coproparasitario en las 49 familiares que convivíar con los lactantes parasitados $y$ que accedieron a participar se muest ra en la Tabla 3. Sólo 4 adultos rehusaron a contribuir. Las madres de los lactantes parasitados tuvieron exámenes negativos excepto una que portaba Giardia lamblia mientras el lactante era portador de Entamoeba histolytica.

Todos los lactantes con infección asintomática tenían un hermano que portaba el mismo enteroparásito que ellos.

La evaluación del estado nutricional en los lactantes parasitados (Tabla 4) es normal en la mayoría de ellos. Desnutrición leve según
Tabla 1.

Detección de parásitos en muestras fecales de 207 lactantes menores de 6 meses.

\begin{tabular}{lrr} 
& $\mathrm{N}$ & $\%$ \\
\cline { 2 - 3 } Presencia de parisitos & 10 & 4,8 \\
Presencia de comensales & 73 & 35,3 \\
Negativos* & 124 & 59,9
\end{tabular}

* Ausencia de parásitos y comensales.

Tabla 2.

Especies parasitarias y comensales encontradas en las heces de 207 lactantes menores de 6 meses.

\section{ESPECIES \\ Enteropatógenas: \\ Giardia lamblia * \\ Entamoeba histolytica** \\ Ascaris lumbricoides*** \\ TOTAL

$\mathrm{N} \quad \%$ \\ Comensales:

\begin{tabular}{lrr} 
Entamoeba coli & 11 & 5,3 \\
Endolimax nana & 71 & 34,3 \\
Chilomastix mesnili & 27 & 13,0 \\
Iodamocba butschilii & 1 & 0,5 \\
\hline TOTAL & 110 &
\end{tabular} \\ * 4 lactantes excretaban G. lamblia mós E. nana; uno G. lamblia más Ch. mesnili y uno G. Jamblia más E. лana e I. butschilii. \\ * In lactante excretaba E. histolytica más Ch. mes- nili. \\ *** Un lactarte excretaba A. lumbricoides más $E$. nana.}

Peso/Edad y Talla $/$ Edad (caso 8) se asoció a Ascaris lumbricoides. Los casos 2 y 10 portado' res de G. lamblia tienen afectados Peso/Taila y Talla/Edad, respectivamente. Varios tenian estos paráme tros por encima de lo normal.

\section{DISCUSION}

Los enteroparásitos han sido descritos pre-

Tabla 3.

Resultado de exámenes coproparasitarios en $\mathbf{4 9}$ pacientes de las familias de lactantes portadores de enteroparásitos.

Negativos
Comensales
Positivos
G. lamblia
E. histolytica
G. lamblia + E. histolytica
A. lumbricoides + G. lamblia
E. histoly tica + vernicularis

\begin{tabular}{rrr} 
& $\mathrm{N}$ & $\%$ \\
\hline & 7 & $(14.2)$ \\
11 & $(22.4)$ \\
& 31 & $(63.4)$ \\
$1 \cdot 8$ & & \\
7 & & \\
4 & & \\
1 & & \\
1 & & \\
\hline & &
\end{tabular}


Tabla 4.

Evaluación del estado nutricional

en lactantes menores de 6 meses parasitados.

\begin{tabular}{clccc}
\hline Caso & Parásito & Peso/Edad* & Talla/Edad** & Peso/Talla*** \\
1 & G. lamblia & 97,1 & 101,7 & 93,2 \\
2 & G. lamblia & 90,0 & 100,5 & 88,7 \\
3 & G. lamblia & 113,7 & 104,8 & 97,4 \\
4 & G. lamblia & 128,2 & 106,5 & 105,3 \\
5 & G. lamblia & 94,2 & 95,6 & 105,8 \\
6 & G. lamblia & 95,5 & 97,2 & 100,7 \\
7 & E. histolytica & 106,8 & 100,2 & 105,3 \\
8 & A. lumbricoides & 86,7 & 92,2 & 100,8 \\
9 & G. lamblia & 106,7 & 96,8 & 116,4 \\
10. & G. lamblia & 112,1 & 93,3 & 115,8 \\
\hline \multirow{2}{*}{ Normal: * $* 90$} & & & \\
$* *$ & $\geqslant 95$ & & &
\end{tabular}

viamente para la población general en Chile ${ }^{2}$ siendo Giardia lamblia y Entamoeba histolytica las más frecuentesmente encontradas. Las tasas de infección general son para Giardia lamblia $16,9 \%$, con las cifras mas altas en la edad de 09 años: $31,2 \%$. Para Entamoeba histolytica son de 18,3 y $8,8 \%$, respectivamente. Los esiudios de portación parasitaria realizados por nosotros en población de bajo nivel socioeconómico y cultu. ral han resultado en cifras todavia mayores a las anteriormente descritas ${ }^{3-4}$.

Se han descrito numerosas variables que inciden en la mayor frecuencia de enteroparásitos en estas poblaciones como son: mal saneamiento ambiental (mala disposición de excretas, ausencia de agua potable o contaminación al acarrearla desde el yrifo hasta que es usada en el interior de la vivienda), presencia de vectores (entre los que se incluyen los animales domésticos), manipula. dores de alimentos que a su vez son portadores de agentes microbiológicos, nivel de escolaridad (que incluye malos hábitos higiénicos), inadecuada recolección de basuras ${ }^{1-9}$. La mayoría de estos factores condicionantes estaban presentes en la población estudiada por nosotros.

Hay escasos estudios de portación en lactantes menores. La Drá. M. Rubio, en $1961^{13}$, realizó una investigación prospectiva en niños hospitalizados: en $24 \%$ de los lactantes menores de un aĩo encontró Giardia lamblia, de éstos $14 \%$ eran menores de 6 meses. En la ciudad de Valdivia ${ }^{14}$ : en 1965-1966 se estudiaron 200 niños ( 18 días a 2 años), asintomáticos, con una tasa de portación para Giardia lamblia de 2,5\%, sin encontrar Entamoeba histolytica. Posteriormente, en 1979, Frenzel y cols. ${ }^{8}$, en la misma ciudad encontracon $12 \%$ y $2,3 \%$ de lactantes menores de 2 años portadores de Giardia lamblia y Entamoeba histolytica respectivamente. En salas-cunas y jardines infantiles de Hospitales de Santiago en $1975^{7}$, la frecuencia de portación de Giardia lamblia fue $17,1 \%$ y de Entamoeba histolytica $0 \%$. en lactantes menores de 1 año. En igual fecha en los exámenes coproparasitarios recibidos en el Hospital Arriarán, provenientes de niños menores de un año las cifras eran de 7,9 y $0,3 \%$, respectivamente ${ }^{15}$. En ninguno de los trabajos mencionados excepto en el de M. Rubio se estudió el grupo menor de 6 meses como tal, lo que fue el objetivo de esta publicación. Además, en la literatura revisada no aparecen estudios de terreno.

Giardia lamblia y Entamoeba histolytica son parásitos frecuentemente asociados a diarrea aguda ${ }^{16-17}$. Ninguno de los sujetos estudiados en esta investigación presentaba esta patología al momento del estudio. en los 15 días antes ni después de tomar las muestras. En consecuencia, se trata de infecciones asintomaticas.

Al menos para bacterias enteropatogenas se ha postulado que la protección otorgada por la leche materna impide el desarrollo de síntomas, sin embargo. no evita la proliferación del agente en el lumen intestinal (infección asintomática) ${ }^{18}$. Los resultados que estamos dando cuenta no pueden ser adscritos a este mecanimno ya que todos los lactantes habían abandonado espontáneamente cl pecho materno.

La excreción fecal de parásitos, bacterias y virus sc ha demostrado en lactantes desdo el periodo de recién nacido en comunidades de mal saneamiento ambiental ${ }^{19}$. Algunas características del agcnte y su interrelación con el huésped $y$ que determinan la presencia de enfermedad han sido descritas y son objeto de activa investigación ${ }^{1,8}$. 
La tasa de portación en este grupo de lactantes menores de 6 meses alçanzó al $4,8 \%$. Esta cifra podría ser todavia mayor si se considera que la técnica de Burrows tiene sólo un $60 \%$ de positividad 20 . Si a la presencia de enteroparásitos se suma la de comensales $(35,3 \%)$ nos indica la alta tasa de contaminación de este grupo etáreo. Ya se ha mencionado que las tasas de portación en el lactante menor de un año alcán. zan 20 a $25 \%$, descontando el $4,8 \%$ de portación en los primeros 6 meses, significa quc la contaminación aumenta aún más durante el segundo semestre de vida. Igual fenómeno hemos observado para bacterias enteropatógenas ${ }^{9}$. Cabria especular que se debiera al incremento del consumo de alimentos sólidos contaminados. en este período de la vida.

Los enteroparásitos descritos pueden afectar cl estado nutricional de los individuos portadores o sintomáticos ${ }^{21}$ y desde este punto de vista su erradicación está indicada. Sin embargo, estudios previos demuestran que para poblaciones con cste nivel de contaminación familiar. el tratamiento debiera alcanzar a todo el grupo lami$\operatorname{liar}^{22}$

Si como se supone, los lactantes sur los más protegidos dentro de la familia, es sorprendente que lactantes menores de 6 meses sean portadores de parásitos y por las razones anunciadas anteriomente, son un problema de Salud Pública que exige lá atención de todos aquellos que trabajan en este campo.

\section{RESUMEN}

Se describe la portación parasitaria y de co. mensales en 207 lactantes menores de 6 meses residentes en área pcriurbana con mal saneamien. to ambiental, y en sus familiares. Se detectó parásitos (G. lamblia. E. histolytica, A. lumbricoides) en el $4,8 \%$ de los lactantes y en $14 \%$ de los familiates. No hay correlación entre el estado nutricional y la presencia de parásitos. En todos los lactantes parasitados se encontró un familiar que portaba el mismo enteroparásito.

\section{AGRADECIMIENTOS}

Este trabajo fue parcialmente financiado por Nestlé Research.

Los autores desean expresar sus sunceros agradecimientos a la Dra. Lucia Toro, Directora; Sra. Inés Riumalló, Enfermera Jefe y al personal del Policlínico La Faena y de terreso, que cooperaron en la realización de esta investigación; a la Srta Julia Abbate, por su labor de secretaría.

\section{REFERENCIAS}

1. Mara, $L .:$ Sociocultural factors in the control and prevention of parasitic discases. Reviews of infectious diseases. 4: 871, 1982 .

2. Schenone, H., Rojas, A., Galdames, M., Villarroel, F.: Aspectos epidemiológicos de las infecciones humanas por protozoos $y$ helmintos intestinales en Chile $(1970-1980)$. Bol. Chile Parasit 36: 44, 1981 .

3. Espinoza. J., Aroyo, M., Pacheco, I., Figueroa, G., Brtunser, $O$.: Infección y reinfección por Giardia Lamblia en escolares aparentemente sanos de nivel socioeconómico bajo en Santiago. XX Reunión Anual de la Sociedad Latinoamericana de Investigación Pediátrica, Lima, Perú, 1982

4. Araya. M., Figueraa, G. Espinoza, J., Zarur, $X$, Brunser, $O .:$ Acutc diarnhoea and carrier state in Chilean pre-schoolers of the low and high socioeconomic strata. Aceptado para publicarse en Acta Paediatrica Scandinavica, 1985

5. Solomons. N.W.: Giardiasis: Nutritional inplications. Reviews of infectious diseases. 4: 859, 1982 .

6. Diamond, L.S.: Amebiasis: Nutritional implications. Reviews of infectious diseases. 4: 843, 1982.

7. Noemi, I., Reyes, H., Mauro, G.: Infecciones por enteroparásitos en rifios de Sala Cuna y Jardín Infantil de dos hospitales de Santiago. Rev. Chil. Pediatr. 46: 50, 1975.

8. Frenzel, A., Torres, P. Guerrero. S., Gesche. P, Montefusio. A., Morin, F.: Parasitosis intestinal en lactances $y$ su relación con la infección de sus manipuladores de alimentos y el saneamiento ambicrtal. Rev. Med. Chile. 107: 343. 1979.

9. Figuerou, G., Troncoso, $M$., Espinoza, J., Arava, $M$, Bustos, M.E., Brunser, $O$.: Portación de entetopatogenos bacterianos en lactantes aparentemente sanos. $X X I l$ Reunión Anual de la Sociedad Latinoamericana de Investigación Pediática. Los Andes. Chile. 1984.

10. National Center for Health Services: Growth curves for children birth to 18 years. United States. Rockville, Md., US.A. National Center for Health Statistics rvital and health statistics. Serie II, No 165 . DHEW publication (PHS) 78-1650). 1977.

11. Alvarez, M.L., Wurgaft, F., Salazar. M.E.: Mediciones de nivel socioeconónico bajo urbano en familias con lactantes desnutridos. Arch. Latinoamer. Nutr. 32: 325,1982 .

12. Burrows, R.B.A.: A new fixative technique for the diagnosis of intestinal parasites. Am. J. Clin. Pathol. 48: 342,1967 .

13. Rubio, $M$.: Encuesta enteroparasitológica en el Hospiral de Niños Luis Calvo Mackenna. en Santiago. Consideraciones clinicas y epidemiológicas sobre 270 casos. Bol. Chile Parasít. 17: 93, 1962

14. Puga, S.: Protozoos $y$ helmintos intestinales en lactantes de la ciudad de Valdivia, Chile. Bul. Chile Parasit. 28: 39.1973

15. Pérez, $C$. Vallejos, E.: Entetoparasitosis intantil en el Area Metropolitana de Salud Central de Santiago. Rev. Chil. Pediatt, 48: 161, 1977.

16. Mata, L.: Epidemiology of acute diarrhoea in childhood: an overview. In: Acute diarhoea: Its nutr. tional consequences in children, edited by J.S. Bellanti. Nestle, Vevey/Raven Press, N.Y. pg. 3, 1983 
17. Araya, M. Brunser, O., Espinoza, J., Figueroa, G., Montesinos, N., Spencer, E.: Etiología de la enfermedad diarreica aguda XIX Reunión Anual de la Sociedad Latinoamericana de Investigación Pediátrica. Quito, Ecuador, 1981.

18. Figuero, G., Troncoso, M., Araya, M., Espinoza, J., Brunser, O.: Enteropathogens carriage by healthy individuals living in a contaminated area with poor sanitation. J. Hyg, Canbridge 91: 499, 1983.

19. Mara, L., Urruria, I.J.: Intestinal colonization of breast-fed childien in a rural afea of low socioeconomic level. Am. N.Y. Acad Sci. 176: 93, 1971.
20. Guiraldes, E. Venegas, G. Gutiérrez, C., Mauro, G., Lotorre, J.J., Noemi, I.: Estudio comparativo de tres roétodos para el diagnóstico de giardiasis. Rev. Med. Chile 110: 21, 1982.

21. Beisel, W.R.: Synergism and antagonism of parasitic diseases in mainutrition. Review of infectious Diseases 4: 746, 1982.

22. Gottlieb, B., Tapin, R., Reyes, H.: Giardiasis familiar: estudio clínico y epidemiológico a partir de casos indices. Rev. Med. Chile 110; 224, 1982. 